\title{
Performance of Channel Inversion Schemes for Multi-User OFDMA
}

\author{
Nicolas Bonneau*, Mérouane Debbah ${ }^{\dagger}$, Are Hjørungnes* and Eitan Altman* \\ *MAESTRO, INRIA Sophia Antipolis, 2004 Route des Lucioles, B. P. 93, F-06902 Sophia Antipolis, France \\ Email: nbonneau@sophia.inria.fr; altman@sophia.inria.fr \\ ${ }^{\dagger}$ Mobile Communications Group, Institut Eurécom, 2229 Route des Crêtes, B. P. 193, F-06904 Sophia Antipolis, France \\ Email: debbah@eurecom.fr \\ *UniK - University Graduate Center, University of Oslo, Instituttveien 25, P. O. Box 70, N-2027 Kjeller, Norway
}

Email: arehj@unik.no

\begin{abstract}
A novel multi-user diversity scheme for OFDMA (Orthogonal Frequency Division Multiplexing Access) is described which alleviates the need of feedback and provides substantial improvements in non-cooperative environments. The algorithm exploits the reciprocity of the channel and enables a user to send reliably data at a prescribed rate knowing only its channel. Moreover, analytical expressions of the cell spectral efficiency are derived in the asymptotic regime (high number of carriers) for two filter types: matched filter and optimum filter. Discussions are also provided for various channel models.
\end{abstract}

\section{INTRODUCTION}

A $N$ carrier OFDM (Orthogonal Frequency Division Multiplexing) system [1] using a Cyclic Prefix (CP) or Zero-Padding [2] for preventing inter-block interference is known to be equivalent in the Frequency Domain (FD) to $N$ flat fading parallel transmission channels. In this paper, we consider a single cell network with $K$ users simultaneously communicating with a base station using OFDM modulation over $N$ carriers (see Figure 1). For this setting, efficient scheduling algorithms to optimize the user's rate exist and are based on multi-user diversity schemes [3] (only users with the best carrier to noise ratio (CNR) conditions access the network). This algorithm requires an estimation by the scheduler (here the best station) of the $N$ carriers of the $K$ users and can increase dramatically the feedback load. Moreover, for high mobility, the channel conditions vary and the algorithm becomes inaccurate. To reduce the feedback load, selective multiuser diversity algorithms have been introduced: only the users that have a CNR above a threshold send feedback to the scheduler [4]. Multiple feedback thresholds can be used [5] and are generally found numerically.

In this paper, we describe a novel multi-user diversity scheme for OFDMA [6], [7] in which no feedback needs to be sent. Namely, each user knows only the channel coefficients of its $N$ carriers whereas the scheduler has no channel knowledge. This is done by exploiting the reciprocity of the channel: A broadcast training sequence is sent by the base station to all the users at the beginning of the communication. Each user estimates its channel and based on an algorithm detailed afterwards selects the carriers ensuring the required data rate. Surprisingly, under mild asymptotic conditions, the algorithm enables each user to send reliably data at a prescribed rate knowing only its channel. For several channel models, we derive analytical expressions of the cell spectral efficiency in the asymptotic regime (high number of carriers) for two filter types: matched filter and optimum filter. Other type of filters such as the MMSE can be studied in the same manner.

In section II, we describe the channel inversion OFDMA model under consideration. Section III provides explicit expression of the spectral efficiency for the matched and optimum filter. Section IV analyzes the Gaussian Model whereas section V-A treats the fading model case. Finally, we draw our conclusions in section VI.

\section{MOdEL}

The scheme considers TDD (Time Division Duplex) mode slotted transmissions on a channel with coherence time $T_{C}=$ $D N T$, where $T$ is the time to transmit one symbol ( $N T$ is the time to transmit one OFDM symbol with $\mathrm{N}$ symbols). At the beginning, the scheduler sends a broadcast training sequence to all the users: it is a known sequence of $G<D$ OFDM symbols. Each user $k$ estimates its $N$ carriers $h_{k}(i), i \in\{1, \ldots, N\}$ and can transmit during the remaining time $(D-G) T$. The spectral efficiency should therefore be reduced by a factor $\frac{D-G}{D}$. In all the following, we will however suppose that the channel is perfectly estimated and $\frac{D-G}{D} \rightarrow 1$.

Constrained on its total power budget $P W$ ( $P$ is the power spectral density and $W$ is the available bandwidth ${ }^{1}$ ), user $k$ selects $L_{k}$ carriers, where $L_{k}$ depends on the particular realization of the fading. On each selected carrier $i$, user $k$ sends the information $x_{k}(i)=\frac{s_{k}(i)}{h_{k}(i)}$, where $s_{k}(i)$ is the transmitted data such as $\mathbb{E}\left[\left|s_{k}(i)\right|^{2}\right]=\frac{P W}{N}$. Therefore, the scheduler does not need to know the channel state information in this "channel inversion scheme" which alleviates the need of a feedback mechanism.

Each user chooses a set $S_{k} \subseteq\{1, \ldots, N\}$ of $L_{k}\left(1 \leq L_{k} \leq N\right)$ carriers such as $\sum_{i \in S_{k}} \frac{P W}{N\left|h_{k}(i)\right|^{2}} \leq P W$. Thus $S_{k} \subseteq\{1, \ldots, N\}$ is

\footnotetext{
${ }^{1}$ Note that in this setting the inter-carrier spacing is $\frac{W}{N}$.
} 
the set of cardinal $L_{k}$ such that $S_{k}$ contains the $L_{k}$ best carriers such as

$$
\frac{1}{N} \sum_{i \in S_{k}} \frac{1}{\left|h_{k}(i)\right|^{2}} \leq 1 .
$$

Note that in this non-cooperative scenario, for each carrier $i$, a set $\mathbb{M}_{i} \subseteq\{1, \ldots, K\}$ of users can select the same frequency carrier $i$, which introduces interference. As a consequence, the received signal on carrier $i$ at the base station is given by:

$$
y(i)=\sum_{k \in \mathbb{M}_{i}} s_{k}(i)+n(i)
$$

where $n(i)$ is a zero mean gaussian noise with variance $\frac{N_{0} W}{N}$.

\section{SpeCtRAL EFFICIENCY}

Let $\operatorname{card}\left(\mathbb{M}_{i}\right)=M_{i}$. Note that $\sum_{i=1}^{N} M_{i}=\sum_{k=1}^{K} L_{k}$. Assuming that each transmitter simultaneously transmits Gaussian-like signals using a different random code book (known by the base station), the spectral efficiency of the cell is given by:

- For the optimum filter:

$$
\gamma_{\text {optimum }}(K)=\frac{1}{N} \sum_{k=1}^{K} \sum_{i \in S_{k}} \frac{1}{M_{i}} \log _{2}\left(1+\frac{M_{i} P}{N_{0}}\right) .
$$

- For the matched filter:

$$
\gamma_{\text {matched }}(K)=\frac{1}{N} \sum_{k=1}^{K} \sum_{i \in S_{k}} \log _{2}\left(1+\frac{P}{\left(M_{i}-1\right) P+N_{0}}\right) .
$$

By optimum, we refer to a joint decoding of all the users with separate code books [8] (or successive stripping of the users where the equivalence is shown in [9]). The matched filter corresponds to the case where all the users (except the user of interest) are considered as background noise. The spectral efficiencies in (3) and (4) are expressed in bits/seconds $/ \mathrm{Hz}$ and are a priori random variables that depend on the realization of the channels. The rates are achievable if the transmitters know exactly these rates. In the following, we will consider the channel in the asymptotic regime (high number of carriers $N$ ) and show that under some assumptions on the channel statistics, the transmitters can send reliably data at a predictable rate irrespective of a particular realization of the interfering channels. Let $\frac{P}{N_{0}}=$ snr; for $M \geq 1$ we define the single-user capacities

$$
\begin{array}{r}
\gamma_{\text {optimum }}^{\text {su }}(M)=\frac{1}{M} \log _{2}(1+M \text { snr }) \\
\gamma_{\text {matched }}^{\text {su }}(M)=\log _{2}\left(1+\frac{\mathrm{snr}}{(M-1) \operatorname{snr}+1}\right) .
\end{array}
$$

Using the fact that $\mathrm{snr}=\gamma_{\mathrm{optimum}}^{\mathrm{su}}(M) \frac{E_{b}}{N_{0}}$ (respectively $\mathrm{snr}=$ $\left.\gamma_{\text {matched }}^{\mathrm{su}}(M) \frac{E_{b}}{N_{0}}\right)$ in these expressions, the single-user capacities are solutions of implicit equations. Concerning the singleuser optimum capacity (5), we remark that if $M \geq 1, x=$ $M \gamma_{\text {optimum }}^{\mathrm{su}}(M)$ is the solution of an implicit equation that does not depend on $M$ :

$$
x=\log _{2}\left(1+x \frac{E_{b}}{N_{0}}\right) .
$$

$x$ is known as the Gaussian single user bound in an AWGN single user transmission. The total spectral efficiencies from (3) and (4) can then be calculated as

$$
\begin{aligned}
& \gamma_{\text {optimum }}(K)=\frac{1}{N} \sum_{k=1}^{K} \sum_{i \in S^{k}} \gamma_{\text {optimum }}^{\mathrm{su}}\left(M_{i}\right) \\
& \gamma_{\text {matched }}(K)=\frac{1}{N} \sum_{k=1}^{K} \sum_{i \in S^{k}} \gamma_{\text {matched }}^{\mathrm{su}}\left(M_{i}\right) .
\end{aligned}
$$

(8) and (9) can be equivalently written

$$
\begin{gathered}
\gamma_{\text {optimum }}(K)=\frac{1}{N} \sum_{i=1}^{N} M_{i} \gamma_{\text {optimum }}^{\mathrm{su}}\left(M_{i}\right) \\
\gamma_{\text {matched }}(K)=\frac{1}{N} \sum_{i=1}^{N} M_{i} \gamma_{\text {matched }}^{\mathrm{su}}\left(M_{i}\right) . \\
\text { IV. GAUSSIAN CASE }
\end{gathered}
$$

Let us first analyze the case of a Gaussian (no fading) uplink multiple access network. The result are useful for comparison purposes with the fading case of section V. For each user $k$ and each carrier $i, h_{k}(i)=1$. Therefore, $S_{k}=\{1, \ldots, N\}$ in the setting of the model. (8) and (9) reduce to:

$$
\begin{gathered}
\gamma_{\text {optimum }}(K)=K \gamma_{\text {optimum }}^{\mathrm{su}}(K)=x \\
\gamma_{\text {matched }}(K)=K \gamma_{\text {matched }}^{\mathrm{su}}(K)
\end{gathered}
$$

In the case of the optimum receiver (for which the complexity increases with the number of users as joint processing is performed), the cell spectral efficiency is independent of the number of users and is equal to the gaussian single user bound. One can show however that for the matched filter, the spectral is a decreasing function of $K$ with:

$$
\begin{aligned}
\lim _{K \rightarrow \infty} \gamma_{\text {matched }}(K) & =\lim _{K \rightarrow \infty} K \log _{2}\left(1+\frac{\mathrm{snr}}{(K-1) \operatorname{snr}+1}\right) \\
& =\frac{1}{\ln (2)}
\end{aligned}
$$

To increase the matched filter spectral efficiency of the system, we suppose that only a certain proportion $\beta$ of the carriers is to be used : in this setting, $S_{k} \subseteq\{1, \ldots, N\}$ is a set of $L_{k}=\beta N$ carriers chosen at random for each user. Since the carriers are chosen at random, the distribution of $M_{i}$ (number of users on carrier $i$ with $1 \leq M_{i} \leq K$ ) is binomial with parameter $\beta$ :

$$
\mathbb{P}\left(M_{i}=M\right)=\left(\begin{array}{c}
K \\
M
\end{array}\right) \beta^{M}(1-\beta)^{K-M}
$$

Using (15), (10) and (11) can be written as

$$
\begin{gathered}
\gamma_{\text {optimum }}(K)=\sum_{M=1}^{K}\left(\begin{array}{l}
K \\
M
\end{array}\right) \beta^{M}(1-\beta)^{K-M} M \gamma_{\text {optimum }}^{\text {su }}(M) \\
=x\left(1-(1-\beta)^{K}\right)
\end{gathered}
$$

and

$$
\gamma_{\text {matched }}(K)=\sum_{M=1}^{K}\left(\begin{array}{l}
K \\
M
\end{array}\right) \beta^{M}(1-\beta)^{K-M} M \gamma_{\text {matched }}^{\mathrm{su}}(M) .
$$


Relation (16) shows that $\beta=1$ is optimum for the optimum receiver. Moreover, the cell spectral efficiency is an increasing function of $K$ that achieves the single user bound (whatever the value of $\beta$ ) at the expense of a decoding complexity. For the matched filter, for a given value of $\frac{E b}{N_{0}}$ and number of users $K$, there is an optimum value of $\beta$ as shown in figure 2. Hence, the reduction in interference (by optimizing $\beta$ ) can more than double the spectral efficiency (see figure 3 ).

\section{FADING CASE}

\section{A. Independent Fading}

In this section we consider that the fading coefficients $h_{k}(i)$ are i.i.d. complex random variables with Gaussian distribution $h_{k}(i) \sim \mathcal{N}(0,1)$. In the asymptotic regime, under the assumption that the users know the channel statistics and the total number of users $K$, we have the following proposition.

Proposition 1: As $N \rightarrow \infty$, the mean spectral efficiency with optimum filter has the following asymptotic expression:

$$
\begin{gathered}
\gamma_{\text {optimum }}(K)=\sum_{M=1}^{K}\left(\begin{array}{l}
K \\
M
\end{array}\right) p^{M}(1-p)^{K-M} M \gamma_{\text {optimum }}^{\text {su }}(M) \\
=x\left(1-(1-p)^{K}\right) .
\end{gathered}
$$

As $N \rightarrow \infty$, the mean spectral efficiency with matched filter has the following asymptotic expression:

$$
\gamma_{\text {matched }}(K)=\sum_{M=1}^{K}\left(\begin{array}{l}
K \\
M
\end{array}\right) p^{M}(1-p)^{K-M} M \gamma_{\text {matched }}^{\mathrm{su}}(M) .
$$

The parameter $p$ is given by:

$$
p=\int_{\mathrm{E}_{1}^{-1}(1)}^{+\infty} e^{-u} d u=\exp \left(-\mathrm{E}_{1}^{-1}(1)\right) .
$$

Here, $\mathrm{E}_{1}$ is the exponential integral defined by: $\mathrm{E}_{1}(t)=$ $\int_{t}^{\infty} \frac{e^{-u}}{u} d u$. Given the channel model, $p$ is therefore a known parameter and is approximately equal to 0.7674 . We observe that (18) and (19) are similar to (16) and (17) with parameter $\beta=p$ : in the asymptotic regime, the uncorrelated fading channel is equivalent to the Gaussian channel with a proportion $\beta=p$ of carriers used.

Proof: We use the ergodicity of $h_{k}(i), i=1 \ldots N$ to show that sums involving $h_{k}(i)$ tend asymptotically to fixed values. As $N \rightarrow \infty$, the sum of inverse of square norms of the complex random variables $h_{k}(i)$ tends to an integral with respect to the distribution of the $\left|h_{k}(i)\right|^{2}$, whose distribution is Chi-Squared with 2 degrees of freedom. Namely,

$$
\frac{1}{N} \sum_{i \in S_{k}} \frac{1}{\left|h_{k}(i)\right|^{2}} \rightarrow \int_{u^{*}}^{+\infty} \frac{e^{-u}}{u} d u
$$

Injecting (21) in (1), we obtain $u^{*}=\mathrm{E}_{1}^{-1}(1)$. Using the fact that

$$
\frac{1}{N} \operatorname{card}\left(S_{k}\right)=\frac{1}{N} \sum_{i=1}^{N} \mathbf{1}_{\left\{\left|h_{k}(i)\right|^{2}>u^{*}\right\}} \rightarrow \int_{u^{*}}^{+\infty} e^{-u} d u
$$

and

$$
p=\lim _{N \rightarrow \infty} \frac{1}{N} \operatorname{card}\left(S_{k}\right)
$$

we obtain $p$ as in (20). According to this analysis, asymptotically it is as if each user chose a set of $p N$ carriers at random. The distribution of $M$ is therefore given by a binomial distribution (15) with parameter $p$ and we obtain (18) and (19) from (10) and (11) as in section IV.

A similar analysis can be conducted for any channel model with i.i.d. fading coefficients.

Figure 4 shows the mean spectral efficiency of the matched filter and the optimum filter for various number of users $K$. Realistic monte-carlo simulations have been also performed for $N=256$. The theoretical curves and the simulated curves for both filters match: in other words, in a finite system, a user is able to send data without knowing the interference generated by other users. It also appears clearly that for the matched filter, the optimum number of users in the cell to be considered is one (result already proved in the downlink case in [10]). As far as the optimum filter is concerned, the spectral efficiency increase is substantial and reaches the Gaussian user bound. Note that one can increase the spectral efficiency of the matched filter by choosing an optimized subset of the carriers as in the Gaussian case of section IV.

\section{B. Totally Correlated Fading}

If the fading is totally correlated on all carriers of each user, then for all $i, h_{k}(i)=h_{k} \sim \mathcal{N}(0,1)$, and $L_{k}$ is a random variable distributed as a rounded version of $N\left|h_{k}\right|^{2}$, which follows a Chi-Squared distribution with 2 degrees of freedom. More specifically,

$$
\begin{aligned}
0 \leq \ell \leq N-1, \mathbb{P}\left(L_{k}=\ell\right) \quad= & \mathbb{P}\left(\frac{\ell}{N} \leq\left|h_{k}\right|^{2}<\frac{\ell+1}{N}\right) \\
& =e^{-\frac{\ell+1}{N}}-e^{-\frac{\ell}{N}} \\
\mathbb{P}\left(L_{k}=N\right) \quad= & \mathbb{P}\left(\left|h_{k}\right|^{2} \geq 1\right)=e^{-1}
\end{aligned}
$$

From the distribution of the $L_{k}$, it is possible to determine the distribution of $M$ via the relations

$$
\begin{aligned}
\mathbb{P}\left(M_{i}=M\right)= & \sum_{\ell_{1}=1}^{N} \cdots \sum_{\ell_{K}=1}^{N} \mathbb{P}\left(M_{i}=M \mid L_{1}=\ell_{1}, \ldots, L_{K}=\ell_{K}\right) \\
& \cdot \mathbb{P}\left(L_{1}=\ell_{1}\right) \cdots \mathbb{P}\left(L_{K}=\ell_{K}\right)
\end{aligned}
$$

and

$$
\begin{aligned}
& \mathbb{P}\left(M_{i}=M \mid L_{1}=\ell_{1}, \ldots, L_{K}=\ell_{K}\right) \\
= & \sum_{\substack{\mathbb{M}_{i} \in\{1, \ldots, K\} \\
\operatorname{car}\left(\mathbb{M}_{i}\right)=M}} \prod_{k \in \mathbb{M}_{i}} \frac{\ell_{k}}{N} \prod_{k \notin \mathbb{M}_{i}}\left(1-\frac{\ell_{k}}{N}\right) .
\end{aligned}
$$

The expectation of the spectral efficiency can then be obtained from (10) or (11).

\section{Effect of the Number of paths}

In this section, we would like to assess the effect of channel correlation on the cell spectral efficiency. We consider the particular case of a multipath channel. The model of the channel is given by

$$
c_{k}(\tau)=\sum_{p=0}^{L-1} c_{p k} \delta\left(\tau-\tau_{p k}\right)
$$


where we assume that the channel is invariant during the time considered. In order to compare channels at the same signal to noise ratio, we constrain the fading coefficients to be complex Gaussian i.i.d. random variables with zero mean and variance $\frac{1}{L}$. Usually, coefficients $c_{p k}$ are supposed to be independent with decreasing variance as the delay increases. In all cases, $\sum_{p=0}^{L-1} \mathbb{E}\left[\left|c_{p k}\right|^{2}\right]=1$. For each user $k$, the coefficients $h_{k}(i)$ are the Discrete Fourier Transform of the fading process. The Fourier transform of $c(\tau)$ is

$$
h_{k}(f)=\sum_{p=0}^{L-1} c_{p k} e^{-j 2 \pi f \tau_{p k}}|\Phi(f)|^{2}
$$

where we assume that the filter $\Phi(f)$ is such that, given the bandwidth $W$,

$$
\Phi(f)= \begin{cases}1 & \text { if }-\frac{W}{2} \leq f \leq \frac{W}{2} \\ 0 & \text { otherwise }\end{cases}
$$

Sampling at the various frequencies $f_{1}=-\frac{W}{2}, f_{2}=-\frac{W}{2}+\frac{1}{N} W$, $\ldots, f_{N}=-\frac{W}{2}+\frac{N-1}{N} W$, we obtain the coefficients $h_{i k}, 1 \leq i \leq$ $N$, as

$$
h_{k}(i)=h_{k}\left(f_{i}\right)=\sum_{p=0}^{L-1} c_{p k} e^{-j 2 \pi \frac{i}{N} W \tau_{p k}} e^{j \pi W \tau_{p k}}
$$

For simplicity sake, the delays are supposed to be uniformly distributed according to the bandwidth

$$
\tau_{p k}=\frac{p}{W}
$$

In figure 5, the spectral efficiency has been plotted versus the number of users at $10 \mathrm{~dB}$ for 2 and 16 paths. Interestingly, for the optimum filter, the spectral efficiency decreases with correlation whereas for the matched filter, the results are completely opposite. As the number of users increases, the difference tends however to disappear.

\section{CONCLUSION}

An OFDMA scheme making use of the reciprocity of the channel to alleviate the need for feedback has been proposed and its performances analyzed. Surprisingly, we show that in a non-cooperative environment with channel fading, a user can send reliable data at a prescribed rate knowing only his channel. The result is based on the predictability of the interference as the number of carriers increases. Moreover, in the case of the matched filter, we show that a judicious choice of the number of carriers can dramatically increase the rate (in comparison with the full use of all the carriers). These results put forward the gain achieved by non-cooperative reciprocal transmissions. In order to assess the performance with respect to fully centralized transmissions, the effect of channel estimation and time-variations should be taken into account.

\section{REFERENCES}

[1] J.A.C. Bingham, "Multicarrier Modulation for Data Transmission: An Idea Whose Time Has Come," IEEE Communications Magazine, vol. 28, no. 5, pp. 5-14, May 1990.

[2] Bertrand Muquet, Novel receiver and decoding schemes for wireless OFDM systems with cyclic prefix or zero-padding, Ph.D. thesis, École Nationale Supérieure desTélécommunications, June 2001.

[3] R. Knopp and P. A. Humblet, "Information Capacity and Power Control in Single Cell Multi-User Communications," in Proceedings of the Int. Conf. on Communications, Seattle, WA, June 1995, pp. 331-335.

[4] D. Gesbert and M-S. Alouini, "How Much Feedback is Multi-user Diversity Really Worth?," in Proceedings of the Int. Conf. on Cоттиnications, Paris, France, June 2004, pp. 234-238.

[5] V. Hassel, M. Slin-Alouini, D. Gesbert, and G. Oien, "Exploiting Multiuser Diversity using Multiple Feedback thresholds," in Proceedings of the IEEE Vehicular Technology Conference, 2005, vol. 1.

[6] Li-Chun Wang and Wei-Jun Lin, "Throughput and Fairness Enhancement for OFDMA Broadband Wireless Access Systems using the Maximum C/I Scheduling," in In Proc. of IEEE VTC, Tokyo, Japan, Sept. 2004.

[7] D. Kivanc, U. Tureli, and H. Liu, "Capacity Improvement for Uplink OFDMA," in Proc. of the 25th Asilomar Conference, Pacific Grove, USA, 2002, pp. 1809 - 1812.

[8] T. Cover and J. Thomas, Elements of Information Theory, Wiley, 1991.

[9] M. Varanasi and T. Guess, "Achieving vertices of the capacity region of the synchronous correlated-waveform multiple-access channel with decision-feedback receivers," in IEEE International Symposium on Information Theory, 1997, p. 270.

[10] Jiho Jang and Kwang Bok Lee, "Transmit Power Adaptation for Multiuser OFDM Systems," IEEE Journal on Selected Areas in Communications, vol. 21, no. 2, pp. 1-10, Feb. 2003.

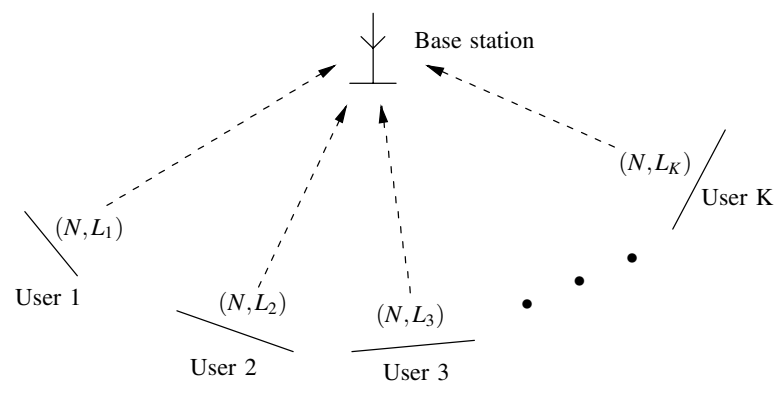

Fig. 1. Multi-User OFDMA Model. 


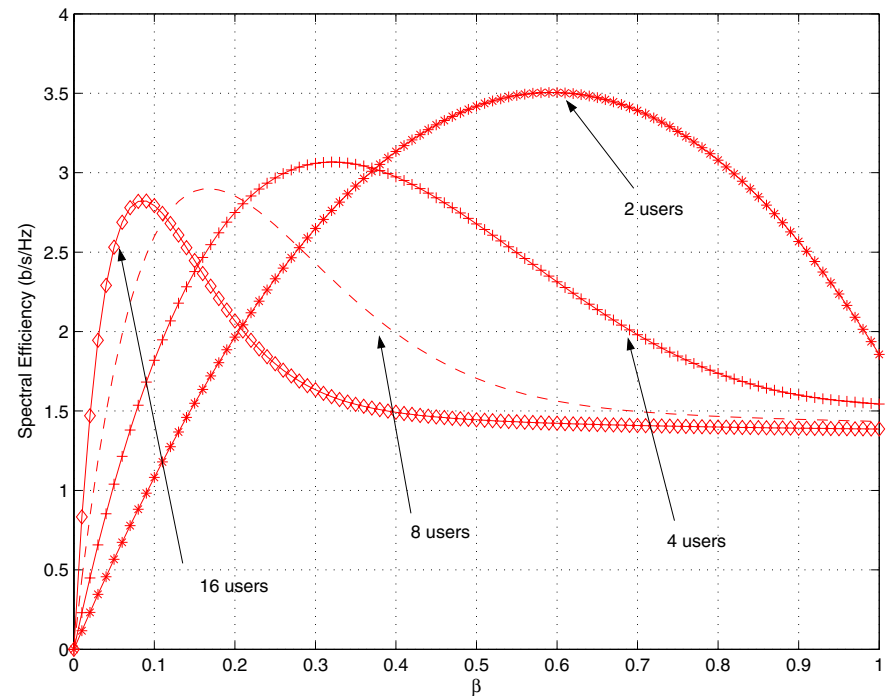

Fig. 2. Spectral efficiency versus $\beta$ for the matched filter in the Gaussian case at $10 \mathrm{~dB}$

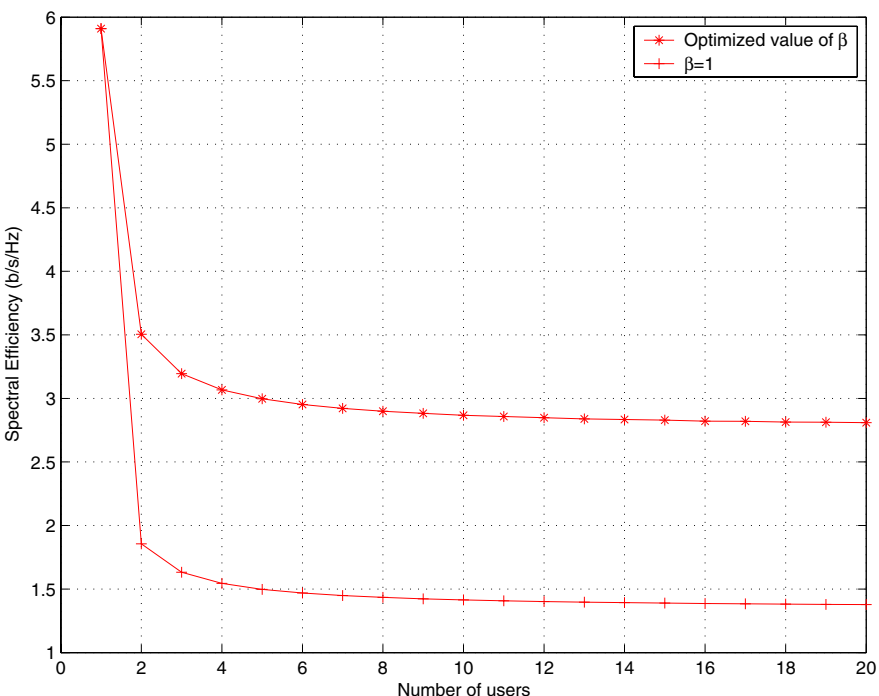

Fig. 3. Spectral efficiency versus the number of users for the matched filter in the Gaussian case at $10 \mathrm{~dB}$

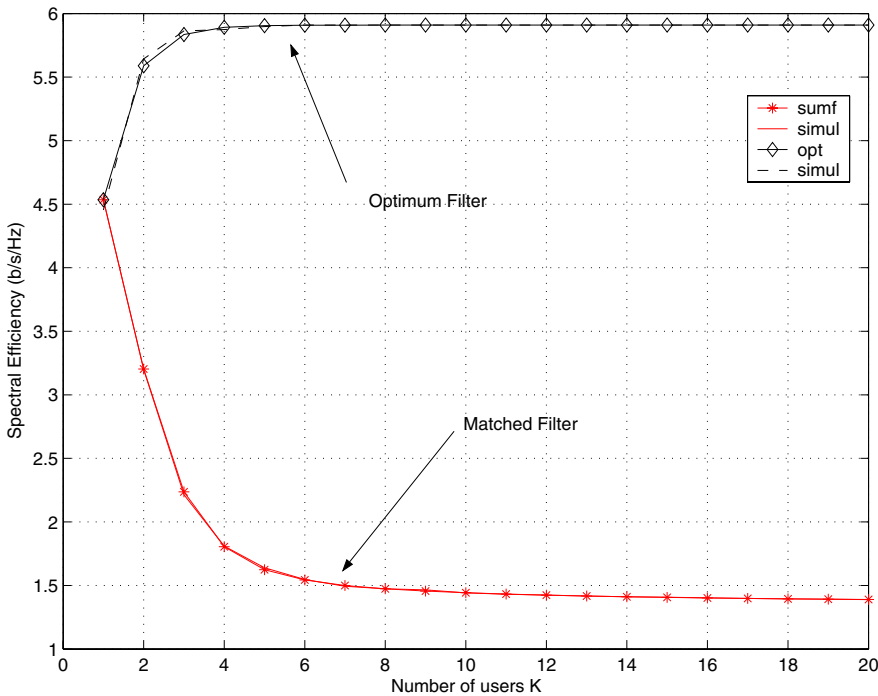

Fig. 4. Spectral efficiency versus the number of users for Rayleigh channel at $10 \mathrm{~dB}$

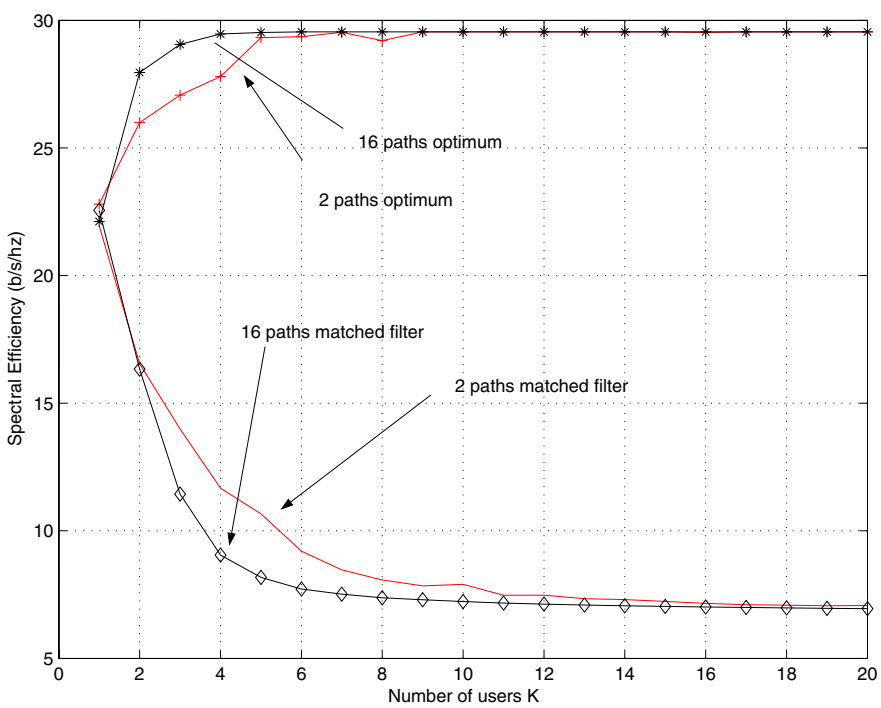

Fig. 5. Spectral efficiency versus the number of users for 2 and 16 paths at $10 \mathrm{~dB}$ 